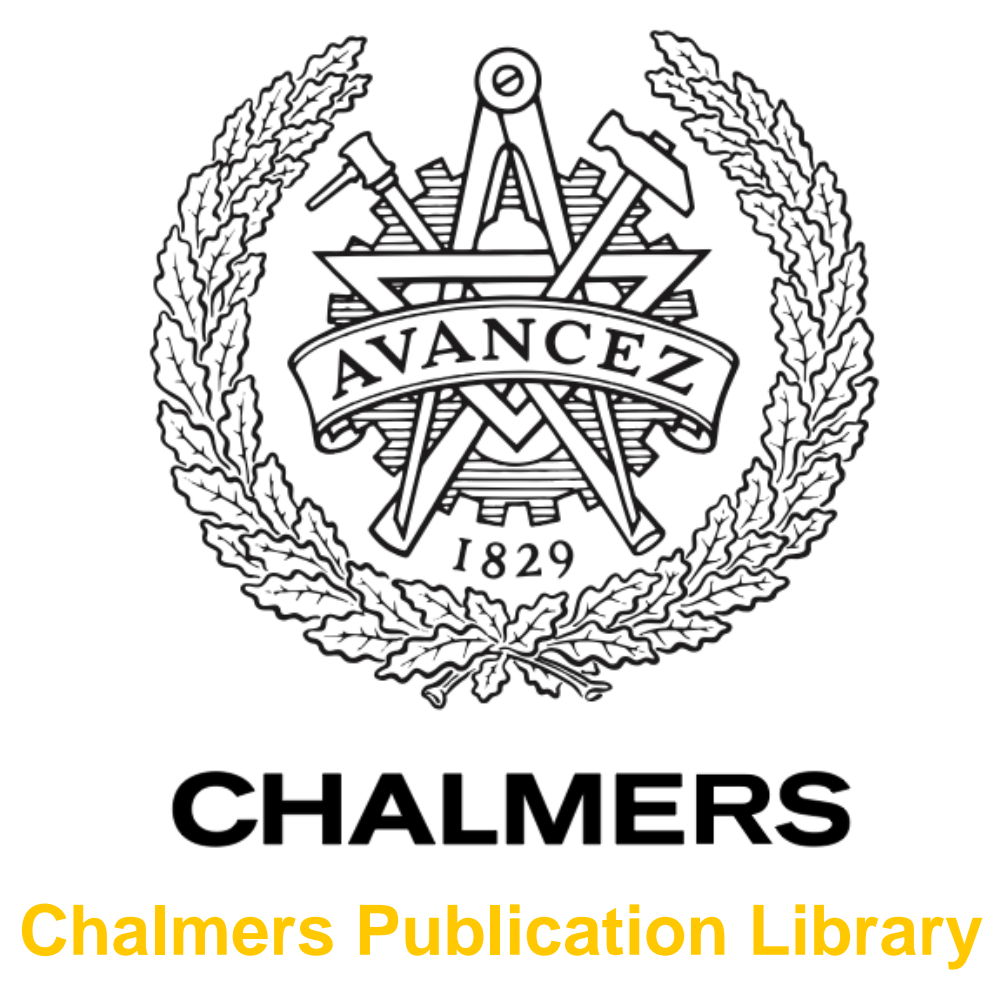

Regenerator site selection in impairment-aware elastic optical networks

This document has been downloaded from Chalmers Publication Library $(\mathrm{CPL})$. It is the author's version of a work that was accepted for publication in:

2016 Optical Fiber Communications Conference and Exhibition (OFC). Anaheim, CA, MAR 20-24, 2016

Citation for the published paper:

Dharmaweera, M. ; Yan, L. ; Karlsson, M. et al. (2016) "Regenerator site selection in impairment-aware elastic optical networks". 2016 Optical Fiber Communications Conference and Exhibition (OFC). Anaheim, CA, MAR 20-24, 2016

http://dx.doi.org/10.1364/OFC.2016.Tu3F.1

Downloaded from: http://publications.lib.chalmers.se/publication/235037

Notice: Changes introduced as a result of publishing processes such as copy-editing and formatting may not be reflected in this document. For a definitive version of this work, please refer to the published source. Please note that access to the published version might require a subscription.

Chalmers Publication Library (CPL) offers the possibility of retrieving research publications produced at Chalmers University of Technology. It covers all types of publications: articles, dissertations, licentiate theses, masters theses, conference papers, reports etc. Since 2006 it is the official tool for Chalmers official publication statistics. To ensure that Chalmers research results are disseminated as widely as possible, an Open Access Policy has been adopted.

The CPL service is administrated and maintained by Chalmers Library. 


\title{
Regenerator site selection in impairment-aware elastic optical networks
}

\author{
Nishan Dharmaweera ${ }^{*}$, Li Yan ${ }^{\diamond}$, Juzi Zhao ${ }^{\diamond}$, Magnus Karlsson ${ }^{*}$, and Erik Agrell ${ }^{\diamond}$ \\ ${ }^{*}$ Photonics Laboratory, Department of Microtechnology and Nanoscience, ${ }^{\triangleright}$ Department of Signals and Systems, \\ Chalmers University of Technology, SE-412 96 Gothenburg, Sweden \\ nishan@chalmers.se
}

\begin{abstract}
We propose a novel regenerator site selection scheme for nonlinear impairmentaware elastic optical networks. The proposed scheme reduces bandwidth use by up to $40 \%$ in a realistic network by employing only 6 regenerator sites.

OCIS codes: (060.4510) Optical communications, (060.4256) Networks, network optimization.
\end{abstract}

\section{Introduction}

Orthogonal frequency division multiplexing (OOFDM)-based elastic optical networks (EONs) increase bandwidth utilization by allocating a contiguous set of subcarriers with "just enough" bandwidth to every connection. In these networks, regenerators are used to counter the detrimental effect of linear and nonlinear physical layer impairments (PLI) and thereby increase the transmission reach of traffic connections. While regenerator site selection in EONs is a relatively new and understudied issue [1-6], a few researchers have attained impressive spectral efficiency gains [1,6], reduced power consumption [3], or reduced bandwidth blocking ratio [4,5] by appropriately selecting regenerator sites. However, these studies do not accurately account for PLI because the transmission reach of their modulation formats was assumed to be independent of the actual network state and because of the use of fixed-size guardbands (GBs) [7].

To overcome these limitations, we propose a novel scheme to identify the appropriate regenerator sites in realistic impairment-aware EONs using the Gaussian Noise (GN)-based PLI model of $[8,9]$. The proposed schemes, which are expressed as an integer linear program (ILP) and a low-complexity heuristic, select the regenerator sites and assign routes, modulation format, and spectrum to offline traffic connections to increase spectral efficiency of the EONs.

\section{Physical-layer impairment model}

The PLI model and values of the relevant parameters are directly adopted from [9]. Let $A_{l}$ denote the set of all connections in link $l$ in a network. For any connection $i \in A_{l}$, the signal-to-noise ratio (SNR) in a single span is $\mathrm{SNR}_{i}=G /\left(G_{\mathrm{ASE}}+G_{\mathrm{NLI}_{i}}\right)$, where $G, G_{\mathrm{ASE}}$, and $G_{\mathrm{NLI}_{i}}$ denote the power spectral density (PSD) of the signal, the amplified spontaneous emission (ASE) noise, and the noise from nonlinear impairments (NLI), respectively. The $G_{\mathrm{NLI}_{i}}$ consists of two terms: self-channel interference (SCI) and cross-channel interference (XCI), where $\mathrm{XCI}=\sum_{j \in A_{l} \backslash\{i\}} \ln \left[\left(\Delta f_{i j}+\Delta f_{j} / 2\right) /\left(\Delta f_{i j}-\Delta f_{j} / 2\right)\right][8,9]$. Here, $\Delta f_{i}$ is the bandwidth of connection $i$ at its assigned modulation format and $\Delta f_{i j}$ is the center frequency spacing between connections $i$ and $j$.

While SCI can be calculated given the connection's bit rate and the spectral efficiency of the assigned modulation format, prior knowledge (i.e., the bit rate, modulation format, and spectrum) of other co-propagating connections is needed to calculate XCI. To obtain this information, every link needs to be closely scrutinized, which is both complex and time-consuming. Thus, we propose an approximation method to estimate the worst case XCI for connection $i$ in a single span. The worst case XCI for connection $i$, which has a bandwidth of $y_{i k}$ using modulation format $k$, occurs when this connection is surrounded by the maximum number of adjacent connections $t$ on both sides, each having the maximum bandwidth of $2 q$, as shown in Fig. 1. In this case, the XCI is $2 \sum_{n=1}^{t} \ln \left[\left(y_{i k}+2 n q\right) /\left(y_{i k}+2(n-1) q\right)\right]$. In this expression, we choose $t$ such that $2 t+1$ equals the highest number of connections in a single link using shortest-path routing for every connection, and $q$ such that $2 q$ equals the bandwidth of the connection with the highest bit rate using the lowest-order modulation format.

Fig. 1: $2 t$ connections surrounding $i$ in a span.

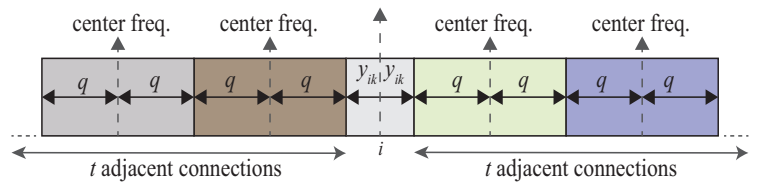

As $G_{\mathrm{NLI}_{i}}$ can now be estimated, the transmission reach for connection $i$ at modulation format $k$ can be calculated in terms of the number of spans as $R_{i k}=\left[\left(G / \mathrm{SNR}^{k}\right) /\left(G_{\mathrm{ASE}_{i}}+G_{\mathrm{NLI}_{i}}\right)\right]$, where $\mathrm{SNR}^{k}$ represents the SNR threshold needed to satisfy the bit error rate (BER) performance of the assigned modulation format $k$. The proposed approximation method provides a more accurate transmission reach estimation than existing work $[1,3,6]$ as it relies on 
the actual bit rate and bandwidth of the connection (without GBs), its assigned modulation format, and the SNR requirement, and as $t$ is based on the traffic distribution in the actual topology.

\section{Problem statement}

Given a network with a set of nodes $V$, a set of links $E$ (all links $l \in E$ have two fibers that carry traffic in opposite directions), and a set of connections $T$, the goal is to identify regenerator sites, perform routing, assign modulation formats, and allocate spectrum to minimize the bandwidth, quantified as the highest subcarrier index $\xi$ assigned to any connection. The transmission parameters are $C$, the spectral width of each subcarrier; $M$, the set of modulation formats; $c_{k}$, the spectral efficiency of modulation format $k \in M ; \Lambda_{i}$, the data rate of connection $i \in T ; \mathrm{SNR}^{k}$, the required SNR threshold of the modulation format $k \in M$; and $R G$, the maximum number of regenerator sites. We denote with $s_{l}$ and $d_{l}$ respectively the source and destination nodes of link $l$, which has a length of $N^{l}$ (or $N^{s_{l}} d_{l}$ ) spans.

The following parameters are predetermined using simple heuristics and the proposed approximation method: $P_{i}$, the set of $K$ shortest routing paths for connection $i \in T ; T_{i k}$, the number of subcarriers required by connection $i$ when assigned modulation format $k \in M$; and $R_{i k}$, the approximated reach of connection $i$ when assigned modulation format $k$. The transparent distance to node $n$ when connection $i$ is routed along path $r \in P_{i}$ is denoted as $W_{i r n}$.

\subsection{ILP formulation}

The proposed ILP aims to minimize $\xi$ by finding the optimal combination of the following binary variables, which are 0 when the condition is not satisfied: $\Psi_{n}=1$ if node $n$ is a regenerator site, $D_{i r}=1$ if connection $i \in T$ is routed along path $r \in P_{i}, p_{i r l}=1$ if connection $i$ travels through link $l$ when routed along path $r, m_{i r l k}=1$ if connection $i$ routed along path $r$ and through link $l$ is assigned modulation format $k, y_{i r j r^{\prime} l}=1$ if connection $i$ that is routed along path $r$ and connection $j \in T$ that is routed along path $r^{\prime} \in P_{j}$ share link $l$, and $u_{i r j r^{\prime} l}=1$ if $y_{i r j r^{\prime} l}=1$ and $f_{i r l}+B_{i r l} \leq f_{j r^{\prime} l}$; and the following integer variables: $B_{i r l}$, the number of subcarriers assigned to connection $i$ on path $r$ and through link $l \in r$, and $f_{i r l}$, the lowest subcarrier index of connection $i$ that is routed along path $r$ and travels through link $l$. The variables should fulfill the following constraints, where $\theta$ is a large inequality coefficient $\left(\theta=10^{5}\right)$.

$$
\begin{aligned}
& \sum_{n \in V} \Psi_{n} \leq R G \\
& \sum_{r \in p_{i}} D_{i r}=1, \quad \forall i \in T \\
& p_{i r l}=D_{i r}, \quad \forall l \in r, r \in P_{i}, i \in T \\
& B_{i r l} \geq \sum_{k \in M} T_{i k} m_{i r l k}, \quad \forall l \in r, r \in P_{i}, i \in T \\
& \sum_{k \in M} m_{i r l k}-p_{i r l}=0, \quad \forall l \in r, r \in P_{i}, i \in T \\
& \xi \geq \quad f_{i r l}+B_{i r l}-1, \quad \forall l \in r, r \in P_{i}, i \in T \\
& \theta \Psi_{n} \geq f_{i r l}-\sum_{z \in P_{i} \backslash\{l\}, s_{z}=n} f_{i r z} \geq-\theta \Psi_{n}, \\
& \quad n=d_{l}, \forall l \in r, r \in P_{i}, i \in T \\
& \theta \Psi_{n} \geq m_{i r l k}-\sum_{z \in P_{i} \backslash\{l\}, s_{z}=n} m_{i r z k} \geq-\theta \Psi_{n}, \\
& \quad n=d_{l}, \forall k \in M, l \in r, r \in P_{i}, i \in T
\end{aligned}
$$

$$
\begin{aligned}
& W_{i r d}=\left\{\begin{array}{l}
W_{i r s}\left(1-\Psi_{s}\right)+N^{l}, \quad l \neq \begin{array}{l}
\text { first link of } r \in P_{i} \\
N^{l},
\end{array} \\
\quad d=d_{l}, s=s_{l}, \forall l \in r, r \in P_{i}, i \in T
\end{array}\right. \\
& \sum_{k \in M} m_{i r l k} R_{i k} \geq \theta\left(p_{i r l}-1\right)+W_{i r d}, \\
& \quad d=d_{l}, \forall l \in r, r \in P_{i}, i \in T \\
& p_{i r l}+p_{j r^{\prime} l} \leq 1+y_{i r j r^{\prime} l} \\
& f_{j r^{\prime} l}+B_{j r^{\prime} l}-f_{i r l} \leq \theta\left(1-y_{i r j r^{\prime} l}+u_{i r j r^{\prime} l}\right) \\
& f_{i r l}+B_{i r l}-f_{j r^{\prime} l} \leq \theta\left(1-u_{i r j r^{\prime} l}\right) \\
& (11)-(13) \text { are valid for } \forall l \in r \cap r^{\prime}, r \in P_{i}, r^{\prime} \in P_{j},\{i, j\} \in T, j \neq i
\end{aligned}
$$

where constraint (1) limits the number of regenerator sites; (2) ensures single-path routing; (3) determines the links that are used on the selected path; (4) and (5) specify the number of subcarriers used by a connection and its modulation format in a link; (6) guarantees that $\xi$ is larger than the maximum subcarrier index of every connection; (7) and (8) allow the spectrum and modulation of a connection to be converted at a regenerator site; (9) determines the distance of every transparent segment; (10) guarantees that the distance in (9) is within the approximated reach of the assigned modulation format; and (11)-(13) guarantee non-overlapping spectrum allocation.

\subsection{Proposed heuristic}

The proposed heuristic involves two stages, the first being the selection of regenerator sites. The preferred routing path for each connection is found using the balance-path-with-heavy-traffic-first algorithm in [10], which is designed to reduce the risk of overloading a single link. For each connection, we first calculate the transmission distance from every node on its preferred path to its destination node. Next, for each node, we sum the remaining transmission distances of all the connections routed via that node. For example, if only connections $i$ and $j$ on paths $\{s-x-d\}$ and $\left\{s-x-w-d^{\prime}\right\}$, respectively, traverse node $x$, the summed transmission distance for node $x$ is $N^{x d}+N^{x w}+N^{w d^{\prime}}$. The nodes are then ranked in descending order of their summed value. The nodes with the highest summed values become the preferred regenerator sites. Lastly, $R G$ number of regenerator sites are assigned in sequence.

In the second stage, spectrum and modulation format are assigned to each connection along every transparent segment of the selected candidate routing path. First, connections are sorted in descending order of their bit rate. Next, the number of subcarriers in each link is initialized to zero (then $\xi=0$ ). Then, starting from the first connection in the sorted list, the connections are served in a sequence. On the candidate path, the length of every transparent segment 
has to be within the approximated reach of the assigned modulation format. Among many possibilities, the candidate path and modulation format that require the lowest subcarrier index is selected for every connection. However, if a connection cannot be routed along any of its candidate paths at any modulation format due to lack of spectrum, the number of subcarriers is increased.

\section{Performance evaluation}

Numerical results are obtained for a small 6-node network (avg. link length $450 \mathrm{~km}$ ), the 14-node Deutsche Telekom (DT; avg. link length $414 \mathrm{~km}$ ) network, the 14-node NSF network (avg. link length $2722 \mathrm{~km}$ ), and the 28-node EU network (avg. link length $1984 \mathrm{~km}$ ) [11]. The parameters required to estimate the PLI take the values given in [7]. Connections are assigned a random bit rate (between A: 0-62.5 Gbps, B: 0-125 Gbps, and C: 0-250 Gbps) in each simulation. The resulting bandwidths in Figs. 2a-2c are the averaged value over ten random $T$.

As Fig. 2a shows that the spectral efficiency of the proposed ILP (when $R G=0$ ) is comparable to that of [7] when the PSD $G<15 \mathrm{~mW} / \mathrm{THz}$, the approximation method provides accurate impairment estimations. Fig. 2b depicts the results for the small 6-node and DT networks, at different traffic volumes, and against an increasing number of regenerator sites. In the 6-node network, it is observed that the heuristic $(\mathrm{H})$, on average, consumes 18-30\% more bandwidth than the ILP. In the DT network, the proposed heuristic reduces $\xi$ by $28 \%, 25 \%$, and $40 \%$ by appropriately placing only 3, 5, and 6 regenerator sites at $\mathrm{A}, \mathrm{B}$, and $\mathrm{C}$ traffic volumes, respectively. The results of Fig. 2c show that the proposed scheme offers higher spectral gains in large networks. In all networks, the spectral gains diminish when the number of regenerator sites in the network is increased beyond a certain threshold. This trend indicates that even with a limited number of regenerator sites, the proposed scheme can achieve spectral efficiency gains, especially in large networks serving a high traffic volume.

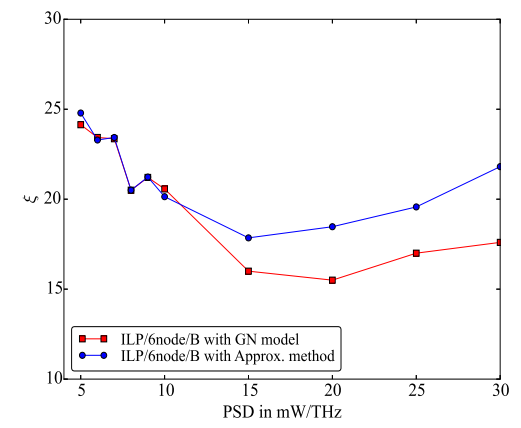

(a)

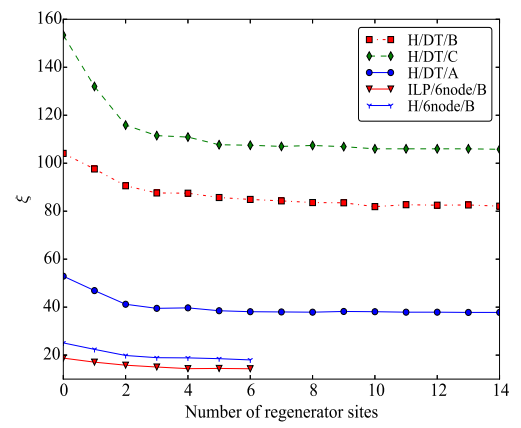

(b)

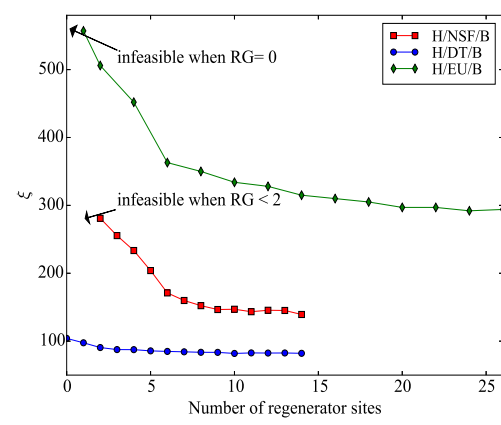

(c)

Fig. 2: $\xi$ vs. (a) PSD $G$ for 6-node network $(R G=0)$, (b) regenerator sites $(G=15 \mathrm{~mW} / \mathrm{THz})$, (c) regenerator sites for DT, NSF, and EU networks $(G=15 \mathrm{~mW} / \mathrm{THz})$.

\section{Conclusion}

The proposed ILP and the heuristic increase the spectral efficiency of impairment-aware EONs by selecting appropriate regenerator sites and by optimizing routing, modulation, and spectrum allocation. The proposed schemes increased spectral efficiency by up to $25-40 \%$ using 3 to 6 regenerator sites at different traffic volumes and in different networks.

\section{References}

1. M. Klinkowski, "On the effect of regenerator placement on spectrum usage in translucent elastic optical networks," in ICTON, Mo.C2.5 (2012).

2. S. Yang and F. Kuipers, "Impairment-aware routing in translucent spectrum-sliced elastic optical path networks," in NOC, (2012).

3. A. Fallahpour et al., "Energy efficient routing and spectrum assignment with regenerator placement in elastic optical networks," JLT, 32, 2019-2027 (2014).

4. M. Aibin and K. Walkowiak, "Adaptive modulation and regenerator-aware dynamic routing algorithm in elastic optical networks," in ICC, 5138-5143 (2015).

5. D. A. R. Chaves et al., "Heuristic algorithms for regenerator assignment in dynamic translucent elastic optical networks," in ICTON, We.B1.2 (2015).

6. X. Wang et al., "Impact of wavelength and modulation conversion on transluscent elastic optical networks using mILP," JOCN, 7, 644-655 (2015).

7. J. Zhao et al., "Nonlinear impairment-aware static resource allocation in elastic optical networks," JLT, 33, 4554-4564 (2015).

8. P. Johannisson and E. Agrell, "Modeling of nonlinear signal distortion in fiber-optical networks," JLT. 32, 4544-4552 (2014).

9. L. Yan et al., "Link-level resource allocation for flexible-grid nonlinear fiber-optic communication systems," PLT, 27, 1250-1253 (2015).

10. C. Xiaojun et al., "A study of waveband switching with multilayer multigranular optical cross-connects," JSAC, 21, 1081-1095 (2003).

11. R. Hulsermann et al., "A set of typical transport network scenarios for network modelling," in 5th ITG Workshop on Photonic Networks, 65-71 (2004). 